\title{
Efficient interactive 3D Livewire segmentation of complex objects with arbitrary topology
}

\author{
Miranda Poon ${ }^{\mathrm{a}, 1}$, Ghassan Hamarneh ${ }^{\mathrm{b}, *}$, Rafeef Abugharbieh ${ }^{\mathrm{a}, 1}$ \\ a Biomedical Signal and Image Computing Laboratory, University of British Columbia, Vancouver, V6T 1Z4 Canada \\ ${ }^{\mathrm{b}}$ Medical Image Analysis Laboratory, Simon Fraser University, Burnaby, V5A 1S6 Canada \\ Received 2 December 2007; received in revised form 10 July 2008; accepted 11 July 2008
}

\begin{abstract}
We present a novel interactive method based on a 3D Livewire approach for segmenting complex objects of arbitrary topologies. Our proposed technique automatically and seamlessly handles objects with branchings, concavities, protrusions, and non-spherical topologies with minimal user-input. Given sparse interactively segmented contours on orthogonal slices, our proposed method determines Livewire seedpoints on all slices in the third orthogonal direction, which are used to mimic user-guided segmentation. In doing so, our method pre-processes these points to increase algorithm robustness, and uses a novel seedpoint sorting method using ideas from L-system's Turtle algorithm. Moreover, we present a segmentation tool based on our proposed framework and demonstrate the robustness of our approach on real medical data. Results highlight the superior performance of the proposed method with validation tests on synthetic and real MRI and CT data, with segmentation reproducibility exceeding $95 \%$ and segmentation task time decreasing to less than $20 \%$ when compared to performing 2 D Livewire on each volume slice.
\end{abstract}

(C) 2008 Elsevier Ltd. All rights reserved.

Keywords: 3D segmentation; Livewire; Interactive segmentation; User-interaction; Medical imaging

\section{Introduction}

In three-dimensional (3D) medical image analysis, segmentation is recognized as vitally important for localizing, quantifying, and visualizing 3D biological structures or other regions of interest. Traditionally, two-dimensional (2D) slicebased manual tracing has been employed, but such a manual operation is very tedious and time consuming because careful delineation is required for each 2D slice within a volume. Also, manually extracted contours viewed from an orthogonal direction typically appear jagged because boundary smoothness is not enforced between slices. Furthermore, manual segmentation methods can suffer from significant inter- and intra-operator variability [1] and user fatigue [2]. Fully automated segmentation techniques, on the other side of the segmentation methods spectrum, work best when their parameters are carefully tuned for specific image properties and anatomy which remains a challenge and still require user validation. An additional compli-

\footnotetext{
* Corresponding author. Tel.: +1 778782 3007; fax: +1 7787823045 .

E-mail addresses: mira.aux@gmail.com (M. Poon), hamarneh@cs.sfu.ca (G. Hamarneh), rafeef@ece.ubc.ca (R. Abugharbieh).

${ }^{1}$ Tel.: +1 6048228851 ; fax: +1 6048225949 .
}

cation factor for these approaches is that anatomical structures are typically affected by significant variations due to subject diversity and pathology which reduces the segmentation accuracy, robustness, and consistency between volumes. Due to the above-mentioned difficulties with both manual and fully automated segmentation techniques, semi-automated methods have drawn wide interest as a way to facilitate computer-based segmentation of 3D anatomical structures using minimal human interaction [2-4]. In this work, we present a novel 3D Livewirebased method for highly automated segmentation of complex objects with arbitrary topology.

A large family of popular segmentation approaches that support or can be extended for user-interaction include parametric, explicit [5,4,6-10], or implicit (e.g. level-set based) [11-13] energy minimizing deformable models. However, these models are prone to convergence to local minima. Active contours that converge to a global minima have been developed [14,15]; however, these rely on a coarser discretization of the search space, succeeded by graph-theoretic optimization procedures that are less amenable to user-interaction. User-interaction in level-set approaches [16] is not straight-forward either, and level-set approaches typically require more computations than other deformable models since contours on 2D images (1D 
manifolds) and surfaces in 3D images (2D manifolds) are represented using a higher dimensional (signed distance transform images), thus increasing the complexity of the problem. Also, employing graphics processing units (GPUs) to perform levelset calculations [17] may be needed to achieve interactivity [18], especially for 3D images. Other recent active contour methods that incorporate user intervention include [19,20]. In Yushkevich et al.'s approach, implemented into the tool called ITK-SNAP [19], user-interaction is used for initializing an evolving 3D active contour, for setting up parameters, as well as for manual post-processing. However, while the initialization is largely graphical, user knowledge of this method's computational part is required for selecting cost parameters, and using imprecise initialization parameters can cause the contour to deviate. Furthermore, the user has no steering control during the curve evolution. In McInerney et al.'s 'Sketch Initialized Snakes' [20], the user uses a graphical tablet to quickly initialize a 2D Snake, which is then automatically optimized. However, this approach requires specialized input hardware. Also, its computational efficiency, reproducibility, and tolerance to user error were not reported.

While interaction with 2D deformable models is more straight-forward, incorporating intuitive user-guidance and realtime visualization for 3D deformable surfaces or meshes remains a challenging human-computer interface problem. One approach to circumvent this problem is to iterate an interactive $2 \mathrm{D}$ algorithm on each slice in the 3D volume [21,22]. However, it is highly inefficient to constantly repeat this task, and without correlating computations within adjacent slices, segmentation results are often jagged (similar to manually delineated ones) when viewed from other orientations. By only requiring sparse user-guided contours or points to dramatically reduce interaction time, there exists many automatic surface reconstruction methods [23,24], some of which can even generate objects with arbitrary topologies [25-27]. However, their main drawback is that they do not consider image-based information; rather, they draw point connectivity and object topology conclusions based on the locations of the surface points alone. Also, their computation time is considerable and does not allow for user intervention during the task should mistakes occur. Similarly, Saboo et al.'s 'GeoInterp' method uses sparsely interleaved manual segmentations in one orientation to initialize a geodesic Snake [28]. However, the user-input is not actually used as a hard constraint, and the Snake optimizes its shape without considering voxel intensities.

An alternative to deformable models is the 'Graph Cuts' approach, originally proposed by Boykov and Jolly [29] and further developed in [30]. Here, 'cuts', or globally optimal segmentations, are computed using manually specified foreground and background seeds (hard constraints) and boundary/region information (soft constraints). Refinement of the 'cut' can be made using additional user-placed seedpoints. This method offers interaction simplicity especially in the 2D case; however, its main drawback is that because the seedpoints lie in the region bodies and not on the boundaries, results can be unpredictable along weak edges. Graph Cuts results will also vary depending on the soft constraint weighting and choice of seeds. Similarly,
Rother et al. proposed the 'GrabCut' method that performs an iterative Graph Cut algorithm that decreases the amount of userinteraction required [31]. Using the same interaction scheme, Grady proposed a method, random walkers, where all pixels in an image are assigned to each of the hard constraint seedpoints based on a probabilistic measure [32]. However, this method still shares the same limitations of the original 'Graph Cuts'. Also, 3D visualization during the segmentation task remains a challenge with this interaction scheme.

Another interactive segmentation paradigm is 2D Livewire (2DLW) [33], which allows direct user-control of the entire delineation boundary. The classical Livewire idea was to break the segmentation problem into segments and have the user guide the formation of each of these globally optimal segments using sparsely spaced seedpoints. The original 2DLW was shown to be consistently accurate [33,34]. To improve its efficiency, numerous modifications such as LiveLane [35] and Livewire on-the-fly $[36,37]$, which limit the algorithm's graph search space, were proposed. While improvements in technology now allow 2DLW to operate in real-time without these modifications, automated Livewire methods that emulate user-input over multiple image slices in a 3D volume still benefit from an abbreviated graph search implementation [38].

In extending Livewire to $3 \mathrm{D}$, several methods requiring only sparse $2 \mathrm{D}$ contours were proposed, but they only consider image slices in one orientation. Souza et al. proposed a hybrid approach between Snakes and Livewire by projecting seedpoints from a previous adjacent slice onto the current slice and then refining their locations [39]. Similarly, Schenk et al. proposed an approach which takes sparsely spaced Livewire contours and interpolates and optimizes the contours in between using minimal cost paths [40]. Also, Malmberg et al. [41] proposed a method to bridge sparsely separated Livewire contours using haptic feedback and the image foresting transform [42]. However, special equipment such as a haptic device and a stereocapable monitor is required. Moreover, image smoothness in orthogonal directions is not ensured, and medical images often contain objects with complex 3D shapes (e.g. deep concavities, protrusions, non-spherical topologies, branching), which none of these parallel-slice approaches are able to effectively handle.

An approach was put forth by Falcao et al. to extend 2D Livewire to 3D by utilizing 2D contours on oblique slices to automatically mimic $2 \mathrm{DLW}$ on all slices in an orthogonal direction [43]. However, considerable user supervision and knowledge regarding the object's exact topological features are required to break a complex object down into 'slabs', which are groups of consecutive slices along the axis of automatic computation where the sub-object exhibits constant topology. The restrictions on these initial setup steps and on the selection of slices for 2DLW are critical to correctly segment each slab properly. Moreover, the intersection of these $2 \mathrm{D}$ contours with each slice in each slab generates seedpoints that need to be manually ordered in a clockwise or counterclockwise fashion before they can be fed into the automated Livewire process. More recent 3D Livewire methods mitigate some of the above setup steps by using orthogonal 2D Livewire contours instead of oblique contours $[44,45]$. In Lu et al.'s 3D Livewire approach [44], seedpoint ordering is 
more automated than in [43], but it requires the projection of a manually supplied reference contour onto adjacent slices. In Hamarneh et al.'s approach [45], seedpoint ordering is automatically computed using an algorithm based on turtle graphics [46] (part of the Logo programming language) without additional image-based or user-supplied information. However, while both of these methods $[44,45]$ do not require the complicated interaction steps of [43], these methods fail to address the problem of segmenting objects of arbitrary topology. Though these semiautomated methods presented reasonable solutions for certain segmentation tasks, their limitations highlight the need for a robust 3D segmentation approach that can natively handle complex shapes of arbitrary topology, while at the same time still offering the advantages of user-control, efficiency, accuracy, intuitive operation, and minimal user supervision.

To address the problems inherent to previous approaches as highlighted above, we propose a novel framework for 3D Livewire-based segmentation that firstly, natively handles arbitrarily complex 3D object geometry and topology in a manner transparent to the user [45,47] (Section 2). Secondly, this method only requires the user to only provide a few sparsely separated contours (no setup tasks as opposed to the slab definition and manual point ordering steps in [43]). Our proposed algorithm can automatically determine Livewire seedpoints on all slices in an orthogonal direction and then segment the object(s) quickly and robustly without further user supervision. The derived seedpoints are valid boundary points because they are a subset of user-approved contours. Thirdly, an interactive and intuitive segmentation tool based on the proposed method is presented. The tool allows for easy transition between 2D and 3D Livewire modes, provides concurrent viewing of contours in all three orthogonal orientations, facilitates efficient correction of user mistakes, and displays 3D visualization of the 2D contours as well as the 3D segmentation result. We validated our proposed method on a variety of synthetic and real life datasets containing objects of different complexities (Section 3). Tests on real medical image volumes show a $95 \%$ rate of reproducibility and an $80 \%$ reduction in task time when compared to performing 2DLW on each volume slice.

\section{Methods}

The user begins the overall segmentation process by performing sparsely separated 2D Livewire (Section 2.1) segmentations on slices in any two orthogonal orientations. Essentially, these contours serve as robust constraints on the overall segmentation process because they are user-guided. These 2D contours are then used to determine the Livewire seedpoints to be used in the third orthogonal orientation (Section 2.2) intersecting the available 2D contours and the unseen orthogonal slices (Section 2.2.1). These intersection points are pre-processed to increase robustness (Section 2.3) and are then used to create a 'turtle map' which consists of orthogonal line segments. Our 'turtle' point ordering algorithm [45] is then applied to this map such that the resulting ordered points mimic the sequence of points a user would select during a semi-automated Livewire segmentation, but now in a fully automated manner on the unseen slices
(Section 2.2.2). Since these new seed points are a subset of the contours previously approved by the user, they are therefore a suitable choice of seedpoints for guiding the Livewire segmentation. In our proposed scheme, user-generated contours that are circumscribed inside another contour are automatically flagged, and such flags are used to split and merge sections of the turtle map (Section 2.5). By doing so, multiple closed contours and objects with non-spherical topologies such as, for example, a vertebra (which has a toroidal topology due to the spinal canal) can be processed correctly. Fig. 1 and Algorithm 1 summarize our approach.

\subsection{Overview of $2 D$ Livewire}

Our proposed method segments 3D objects based on the use of 2DLW (though in orthogonal orientations) to compute some sparse initial contours for further automated segmentation. 2D Livewire is a widely known interactive segmentation technique where sparse seedpoints are specified along an object's boundary and the path between the points is optimized using dynamic programming [33]. Since the focus of this paper is not traditional 2D Livewire, we only provide a brief explanation of the optimization costs we used. For a detailed analysis of Livewire's implementation, we draw interested readers to [33]. In particular, Fig. 1 in [33] walks through the optimization mechanism and optimal path determination for a small sample space.

In an $X Y$ slice $S(q)$ of a volume (similarly for $X Z$ and $Y Z$ slices), where $q=\left(x_{1}, y_{1}\right)$, image edge and contour smoothness terms are used to create a local cost map $C(p, q)$ of the original image (1). Here, $p=\left(x_{2}, y_{2}\right)$ represents a neighboring pixel to pixel $q$. In our implementation, the gradient magnitude cost $C_{\mathrm{G}}(q)$, gradient direction cost $C_{\mathrm{GD}}(p, q)$, Canny edge detection [48] cost $C_{\mathrm{C}}(q)$, Laplacian of Gaussian (LoG) cost $C_{\mathrm{LoG}}(q)$, and Euclidean distance (smoothness) cost $C_{\mathrm{d}}(p, q)$ were used as follows:

$$
\begin{aligned}
C(p, q)= & w_{1} C_{\mathrm{C}}(q)+w_{2} C_{\mathrm{LoG}}(q)+w_{3} C_{\mathrm{G}}(q) \\
& +w_{4} C_{\mathrm{GD}}(p, q)+w_{5} C_{\mathrm{d}}(p, q) .
\end{aligned}
$$

$w_{1 \ldots .}$ are scalar weights and $C_{\mathrm{G}}(q)$ is defined as

$$
C_{\mathrm{G}}(q)=1-\left.\frac{1}{\max (G)} \sqrt{\left(\frac{\mathrm{d} S(x, y)}{\mathrm{d} x}\right)^{2}+\left(\frac{\mathrm{d} S(x, y)}{\mathrm{d} y}\right)^{2}}\right|_{(x, y)=q},
$$

$\max (G)$ denotes the largest gradient magnitude found in the image. The gradient direction cost is then:

$C_{\mathrm{GD}}(p)=\frac{1}{\pi} \arccos \left(\frac{S^{\prime}(p) \cdot S^{\prime}(q)}{G(p) G(q)}\right)$,

where $G(p)$ and $G(q)$ denote the gradient magnitude (not gradient cost) of the image at pixel $p$ and $q$, respectively. The LoG cost of the image is defined as

$$
C_{\mathrm{LoG}}(q)=1-\left.\left(\operatorname{LoG}_{\mathrm{kernel}}(x, y) \cdot S\right)\right|_{(x, y)=q},
$$


(a)

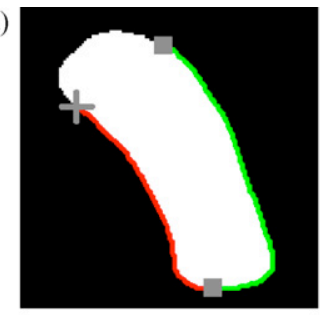

(e)

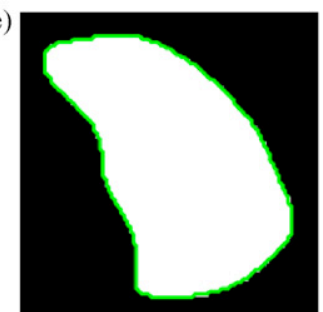

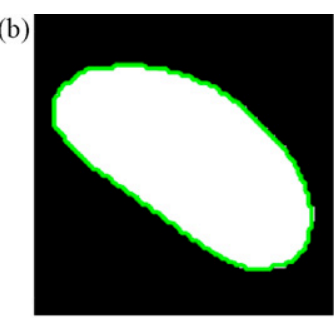

(f)

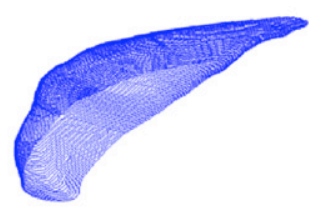

(c)

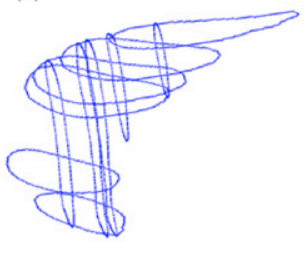

(g)

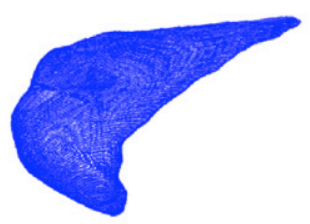

(d)

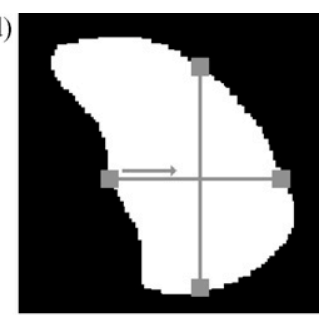

(h)

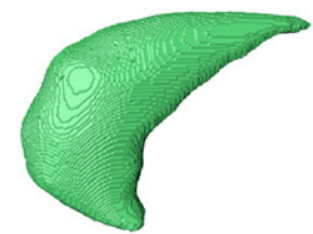

Fig. 1. Overall steps of our proposed algorithm, shown on a binary image for clarity. (a and b) Seedpoints (gray squares) are selected in user-guided Livewire contours on orthogonal slices. User-approved segments are in green, and red contours represent the proposed, 'live' segment during the Livewire task (crosshair denotes cursor location). (c) 3D plot of 11 user-guided contours. (d) Ordering automatically generated seedpoints (gray squares) on a slice in the third orthogonal orientation using our turtle-based algorithm (Section 2.2.2). (e) Result of mimicking Livewire in an automatic fashion using (d). (f and g) 3D plots of automatically generated contours at mid-task and at task completion (125 contours), respectively. (h) Surface rendering of the segmentation result. (For interpretation of the references to color in this figure legend, the reader is referred to the web version of the article.)

where $S$ is the original image and is convoluted with the LoG kernel:

$\operatorname{LoG}_{\text {kernel }}(x, y)=-\frac{1}{\pi \sigma^{4}}\left(1-\frac{x^{2}+y^{2}}{2 \sigma^{2}}\right) \mathrm{e}^{-\left(\left(x^{2}+y^{2}\right) / 2 \sigma^{2}\right)}$.

Lastly, $C_{\mathrm{d}}(p, q)$ is a scalar cost that is proportional to Euclidean distance $\sqrt{\left(x_{1}-x_{2}\right)^{2}+\left(y_{1}-y_{2}\right)^{2}}$.

With a user-specified seedpoint located at pixel $q=\left(x_{1}, y_{1}\right)$ on the slice, the cost map $M(q)$ is then created by determining the minimal accumulated path cost from $q$ to all other pixels on the slice using Dijkstra's algorithm [49]. Although this graph search algorithm is computationally expensive, it is only required once per seedpoint. Using this cost map, the minimal path from an arbitrary point $r=(x, y)$ (mouse cursor location) back to the seedpoint $q$ is continuously found and displayed in real-time [33] as the user freely moves the mouse. Starting at point $r$, the next contour point in the optimal contour is determined by choosing the pixel neighbor with the lowest accumulative cost. As a result, the original seedpoint $q$, with accumulative cost $C_{q}=0$ will always be found. Using this scheme, the user can quickly and easily determine the optimal $r$ to use as the next seedpoint and repeat this process (i.e. $q \leftarrow r$ and a new $r$ is sought) until the contour is closed.

\subsection{Livewire in $3 D$}

The basic idea of 3D Livewire is to use the sparse contours gathered by 2DLW to segment the entire 3D object. Specifically, this algorithm uses the 2DLW contours in two orthogonal directions in order to generate seedpoints for an automated version of 2D Livewire in the third orthogonal direction. These seedpoints, however, must be ordered such that the sequence mimics the order in which a user would select these points. This ordering is important and is explained in detail in Section 2.2.2.

\subsubsection{Automatic seedpoint generation}

Initially, the user performs 2D Livewire on select slices in any two orthogonal orientations (e.g. $\{y z, x z\})$ and an automatic Livewire segmentation can then be performed on a slice in the third orientation (e.g. $x y)$, and similarly for $(\{y z, x y\}, x z)$ and $(\{x z, x y\}, y z)$. These intersection points are simply the intersection between the 2D Livewire contours and the unvisited orthogonal slice. For example, if 2D Livewire is used to create two contours $C_{x_{0} y z}$ and $C_{x y z_{0}}$ on arbitrary $y z$ and $x y$ slices, respectively, then given a slice $S_{x, y_{0}, z}$, in the $x z$ orientation at index $y_{0}$, the intersection points $I_{x, y_{0}, z}$ between $C_{x_{0} y z}$ and $S_{x, y_{0}, z}$, and $J_{x, y_{0}, z}$ between $C_{x y z_{0}}$ and $S_{x, y_{0}, z}$ can be calculated as

$$
I_{x, y_{0}, z} \Leftarrow C_{x_{0} y z} \bigcap S_{x, y_{0}, z}, \quad J_{x, y_{0}, z} \Leftarrow C_{x y z_{0}} \bigcap S_{x, y_{0}, z} .
$$

Similarly, (7) and (8) define the intersection points $I$ and $J$ on slice $S$ if different orientation combinations are chosen:

$$
\begin{aligned}
& I_{x, y, z_{0}} \Leftarrow C_{x z} \bigcap S_{x, y, z_{0}}, \quad J_{x, y, z_{0}} \Leftarrow C_{y z} \bigcap S_{x, y, z_{0}} \\
& I_{x_{0}, y, z} \Leftarrow C_{x y} \bigcap S_{x_{0}, y, z}, \quad J_{x_{0}, y, z} \Leftarrow C_{x z} \bigcap S_{x_{0}, y, z}
\end{aligned}
$$

Fig. 2 summarizes this determination mechanism.

\subsubsection{Point ordering using L-system's turtle}

Mimicking a user-guided 2D Livewire segmentation task in an automatic fashion requires not only the seedpoint locations, but also a clockwise or counterclockwise point ordering. For example, let $I$ and $J$ be seedpoints in the 2D space of slice $S$. Seedpoints on $S$ which belong to the same user-guided Livewire contour subset are paired and connected by lines (tracks). Since there are seedpoint contributions from two orthogonal orientations, these tracks will themselves be orthogonal on slice $S$. Algorithm 1 summarizes this step and Fig. 3 illustrates the final result, called a 'turtle map'. 

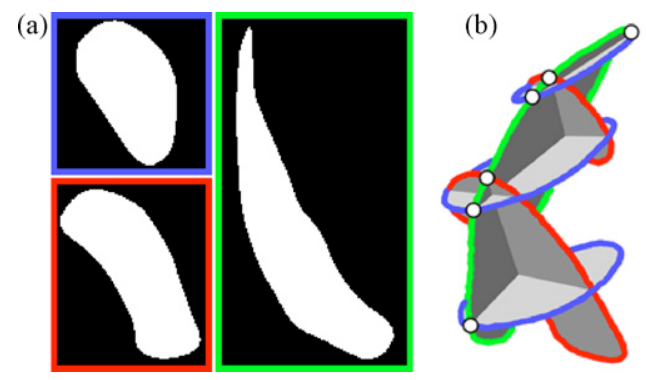

(c)

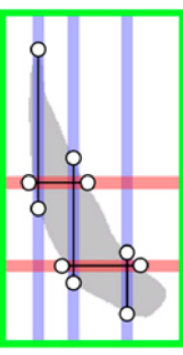

Fig. 2. Orthogonal contours in (a) (red and blue) intersect with a slice in the third orientation (green) in 10 different locations, as shown in (b). These intersections on the green slice in (c) become seedpoints on a turtle map (Section 2.2.2). (For interpretation of the references to color in this figure legend, the reader is referred to the web version of the article.)

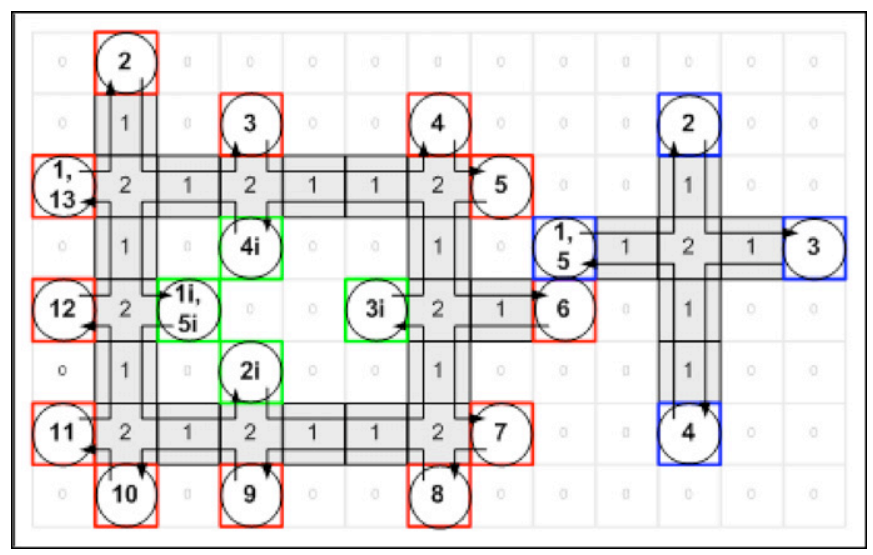

Fig. 3. Example seedpoint map showing outer contour seedpoints (red), inner contour seedpoints (green with 'i' suffix), and a disjoint object's seedpoints (blue). For each contour, the turtle object starts at the first point and follows the tracks (in gray) according to its rules in Section 2.2.2, visiting other seedpoints in the order shown. Track values of ' 2 ' denote track intersections. (For interpretation of the references to color in this figure legend, the reader is referred to the web version of the article.)

Algorithm 1. Determining the segmentation for unvisited slice $S_{x, y_{0}, z}$ in $x z$ given $M$ input contours $\left\{C_{x_{m}, y, z}\right\}$ in $y z$ and $N$ input contours $\left\{C_{x, y, z_{n}}\right\}$ in $x y$. Note that it is a trivial change to process unseen slices in $x y$ and $y z$. (a)

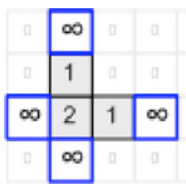

(b)

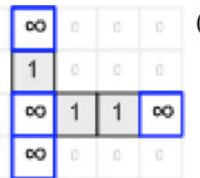

(c)

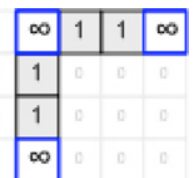

Fig. 4. Possible turtle map intersections resulting from seedpoint locations, denoted by $\infty$, include (a) the basic ' + ' junction, (b) the ' $T$ ' junction where a seedpoint overlaps a track, and (c) the ' $\mathrm{L}$ ' junction where two seedpoints overlap. Pixels with values ' 1 ' and ' 2 ' represent non-seedpoint tracks and track intersections, respectively.

ming language, and its main idea surrounds a directional turtle object that can only move forward and change directions on a graph-based system. Here, our turtle object begins at an arbitrary seedpoint and moves forward along the orthogonal tracks, turning to its left if it encounters a track intersection and reversing direction when it encounters another seedpoint. The sequence in which the seedpoints are visited determines their order (Fig. 3). This process is repeated if there are multiple closed contours found on the same unseen slice, and our method keeps track of which seedpoints have been visited so that they are not encountered again.

While turtle map tracks usually intersect in a ' + ' like shape (Fig. 4(a)), oftentimes turtle maps can exhibit ' $\mathrm{T}$ ' junctions (Fig. 4(b)) and 'L' junctions (Fig. 4(c)), as determined by how the user-guided contours intersect with this unseen slice. Here, our proposed turtle algorithm detects these situations and resolves them correctly by altering the turtle's movement rules and ensuring these seedpoints are not duplicated in the resulting seedpoint list.

\subsection{Intersection point pruning}

To improve robustness of our algorithm in real medical images, a pruning step is taken during the determination of seedpoints in Section 2.2.1. Ideally, a 2D contour would intersect with an unvisited orthogonal slice at an even number of locations, described by Hamarneh et al. as 'entering and exiting' the object [45]. However, objects with cusps can cause singular intersection points to exist as well. Also, while a user-guided contour

Require: $\left\{C_{x_{m}, y, z}\right\}$ and $\left\{C_{x, y, z_{n}}\right\}$ on slices $S_{x_{m}, y, z}$ and $S_{x, y, z_{n}}, x_{m}$ and $z_{n}$ represent arbitrary slice indices of $x$ and $z$ respectively.

Ensure: Closed contours $\left\{C_{x, y_{0}, z}\right\}$ on slice $S_{x, y_{0}, z}$

TurtleMap $\Leftarrow 0$. TurtleMap is the $2 \mathrm{D}$ area on which turtle tracks are placed.

TurtleMap $\Leftarrow$ Algorithm $2\left(\left\{C_{x_{m}, y, z}\right\}, S_{x, y_{0}, z}\right)$, building horizontal tracks of TurtleMap.

TurtleMap $\Leftarrow$ TurtleMap + Algorithm $2\left(\left\{C_{x, y, z_{n}}\right\}, S_{x, y_{0}, z}\right)$, building vertical tracks of TurtleMap.

$P_{\text {ord }}^{a}(x, z) \Leftarrow$ TurtleMap $(x, z)$, ordering seedpoints using Turtle algorithm (Section 2.2.2). $A=$ number

of separate contours on $S_{x, y_{0}, z}$ ), where $A=\max (a)$.

for each $P_{o r d}^{a} \in(1 . . A)$ do

$C_{x, y_{0}, z}^{a} \stackrel{\text { ord }}{\Leftarrow} P_{\text {ord }}^{a}$, using abbreviated graph search (Section 2.4).

end for

$\left\{C_{x, y_{0}, z}\right\} \Leftarrow\left\{C_{x, y_{0}, z}^{a=1}, C_{x, y_{0}, z}^{a=2}, C_{x, y_{0}, z \cdots}^{a=3}\right\}$, all contours with $y=y_{0}$ are found.

The last step to ordering the seedpoints is to employ an algorithm to traverse this map, sequentially visiting each seedpoint. To accomplish this, an algorithm based on turtle graphics [46] was developed. Turtle graphics is based on the Logo program- will always be orthogonal to the slice in question, contiguous colinear contour pixels may intersect with this slice [44]. In the extreme example of a cube, a user-guided contour (a square) may be orthogonal to an unseen slice, but their intersection may 

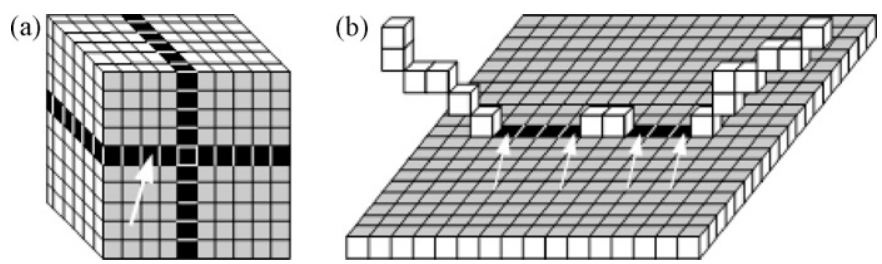

Fig. 5. Contiguous intersection points. (a) User-guided Livewire contours (black voxels) will intersect with the cube's end slice (gray) in multiple contiguous locations. (b) A contour intersecting with an orthogonal image slice (gray) creates two clusters of contiguous points (black). White arrows denote which pixels are kept after our pruning algorithm (Section 2.3).

comprise the entire square side of the contour (Fig. 5(a)). To combat this, our proposed method assumes intersection points appear in cluster(s) or occupy consecutive pixel locations such as in Fig. 5. Since the intersection points found between each user-guided Livewire contour and the orthogonal unvisited slice will always be a horizontal or vertical line on the slice, these colinear points are first sorted in ascending pixel location order. Next, by traversing these points, cluster boundaries are easily found by determining the non-consecutive pixel location values. Knowing where clusters start and end allows us to prune the unnecessary points in between. An exception to the rule is when only one cluster is found, which corresponds to a cusp (singular point). In this case, the start and end of the cluster are kept and the middle points are discarded. With the extraneous contiguous points removed, the desired case of having an even number of intersection points is achieved. This allows for each automatically processed slice to be independent of all other parallel slices and to not require a reference frame [44]. Therefore, shape and topology changes (e.g. branches, cusps, saddle points) not observed in adjacent slices can now be seamlessly detected without further user supervision.

\subsection{Efficient graph search for pre-determined seedpoints}

With a set of sequential seedpoints per unvisited slice, userguided Livewire is easily mimicked in an automated fashion. Since the seedpoints determined from orthogonal contours are pre-determined, no user-interaction is required and thus, an exhaustive 2D search using Dijkstra's algorithm for each seedpoint is redundant. Our solution to this is similar to that in [36], as our modified graph search algorithm terminates after the next target point in the ordered list of seedpoints has been reached. The computational savings originate from the order in which the graph search propagation is done: the propagation algorithm selects the unprocessed pixel with the lowest accumulative cost to be analyzed next [33]. For example, when the graph search propagates from seedpoint $q$ to point $r$, the accumulative cost of $r$ is $C_{r}$. At this point, all arbitrary pixels $p$ with accumulative cost $C_{p}<C_{r}$ would have been found already; thus the path from $r$ back to $q$ is guaranteed to be globally optimal. Fig. 6 illustrates the impact of this technique. The graph search algorithm favours propagation along high-gradient edges and will largely ignore homogenous regions because seedpoints tend to be on or very close to gradient edges. Another advantage is that the computational savings now depend on the distance between seedpoints and not image resolution.

\subsection{Handling arbitrary topology}

Analyzing anatomical structures that exhibit non-spherical topologies, concavities, or protrusions is difficult without first applying a robust segmentation method that can handle any combination of these object conditions. In the simpler case of convex objects (e.g. sphere), it is guaranteed that there will only be one or two clusters of seedpoints per input contour in unseen slices; thus, a turtle map can be easily generated using the technique described in our previously proposed framework [45]. However, for objects with concavities or protrusions (e.g. U-shaped tube), there may be situations where a slice captures multiple objects and its turtle map will show multiple disjoint groups accordingly (Fig. 3). Since our current approach processes each group independently, multiple objects can be segmented concurrently, such as the left and right ventricles (Fig. 9(a)). More complicated still are objects with non-spherical topology (e.g. torus), which none of the previous Livewire methods can handle. In order to correctly segment these objects, our method first identifies contours that are circumscribed within another using pairwise comparisons on all user-guided contours of a given image slice. Let $C_{1}$ and $C_{2}$ represent two closed contours on the same slice. $C_{1}$ and $C_{2}$ are first converted to binary masks $M_{C_{1}}(x, y)$ and $M_{C_{2}}(x, y)$, respectively, where pixels inside the contour have a value of 1 and 0 otherwise. If $M_{C_{1}}(x, y) \bigcap M_{C_{2}}(x, y)=M_{C_{1}}(x, y)$, then $C_{1}$ is wholly situated inside $C_{2}$, and if $M_{C_{1}}(x, y) \bigcap M_{C_{2}}(x, y)=$ $M_{C_{2}}(x, y)$, then $C_{2}$ is wholly situated inside $C_{1}$. This step is critical because these 'inner' contours delineate pixels that do not encompass the object of interest, but rather a hole in the object. Due to this, these contours and their derived seedpoints (Section 2.2.1) are flagged as 'negative', whereas the contours and seedpoints that actually delineate the object are flagged as 'positive'. Both 'positive' and 'negative' intersection points are used on the turtle map; however, turtle tracks are only constructed between 'positive' seedpoints. In contrast, 'negative' seedpoints in effect
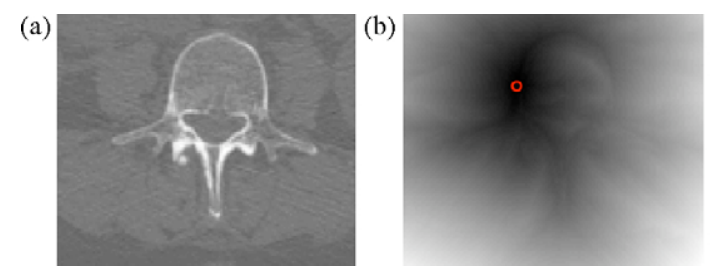

(c)

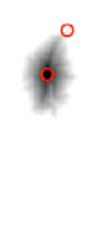

(d)

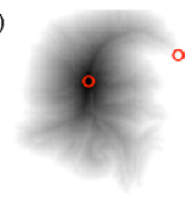

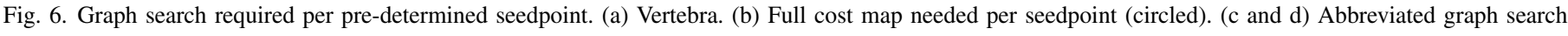
algorithm terminates when the next pre-determined seedpoint is reached. 
negate a section of an otherwise longer track line, splitting the turtle map into two distinct parts. This process is illustrated in Fig. 3, where seedpoints $2 i$ and $4 i$ negate the otherwise longer turtle track between seedpoints 3 and 9 . A central cavity results, which now correctly represents the toroidal object. This process is outlined in Algorithm 2.

Algorithm 2. Constructs the horizontal tracks in TurtleMap that is used for ordering the seedpoints found in Algorithm 1 for an unvisited slice $S_{x, y_{0}, z}$. For vertical tracks and if orthogonal unvisited slices $S_{x_{0}, y, z}$ or $S_{x, y, z_{0}}$ are used, only trivial changes are required. cations. While our technique is flexible and robust, errors are bound to occur due to human error and poor image quality. Our tool offers the undo operation described above, as well as the ability for users to remove entire automatically generated contours for re-computing. From the rendered result, users can quickly identify problematic areas, if any, and increase the segmentation accuracy by providing additional user-guided contours in these areas and re-running the 3D Livewire algorithm. For isolated refinement, users can also choose to overwrite the automatically generated contour(s) using the 2D Livewire procedure.

Require: An empty or incomplete TurtleMap array, $G$ contours $C_{x_{m}, y, z}^{g}$ on slices $S_{x_{m}, y, z}$ respectively. $x_{m}$ represents arbitrary slice indices of $x$.

Ensure: Construction of horizontal TurtleMap tracks.

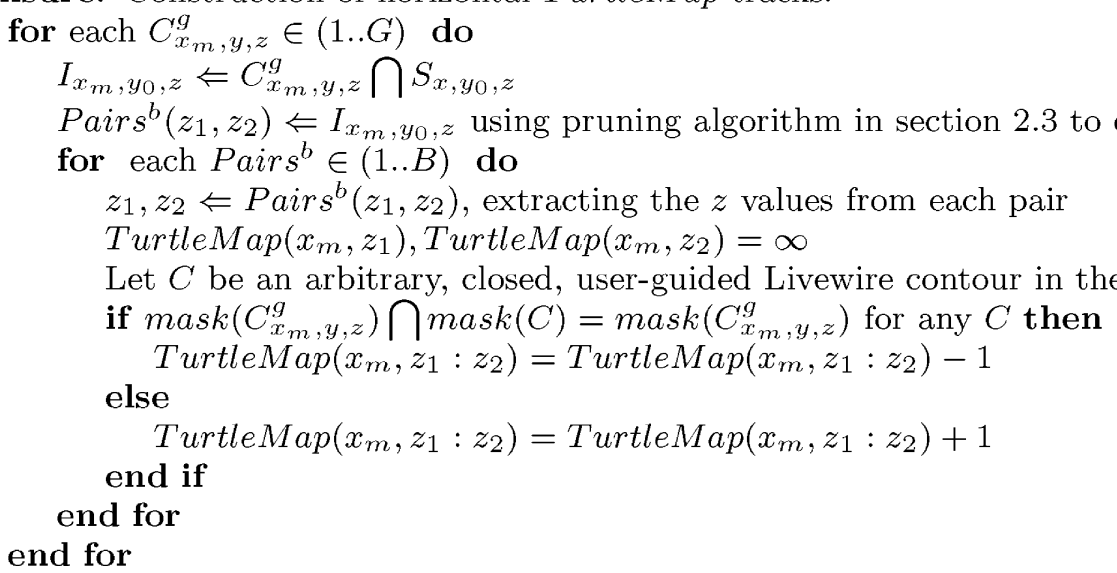

\subsection{Implementation and visualization}

For deployment of this method in clinical settings, our method had a goal of having a high degree of visual interaction and intuitive operation. This proposed framework was developed in MATLAB (The MathWorks Inc., Natick, MA) and offers the standard concurrent orthogonal views of a volume as shown in Fig. 7. As an overlay on top of the image data, user-guided contours are clearly demarcated in all views, regardless of their orientation. One criticism of this type of 3D Livewire extension [45] was that the slices used for user-guided contours had to be carefully selected otherwise the segmentation will fail [44]. By displaying these contours in this manner, our application gives users a clear idea of which areas have been segmented and which areas exhibit more topological features. In our findings, these feature-rich areas, if segmented correctly by the user, usually allow for higher accuracy. Also, this software feature is useful for visually judging the accuracy of the delineation result. Additionally, our user interface is able to display 3D plots of contours as well as a surface rendering of the object of interest after the 3D Livewire procedure is completed.

In our tool, additional features such as point deletion and automatic contour closing are available during the user-guided Livewire stage. Also, if the user selects a seedpoint erroneously, he/she can revert the segmentation process to an earlier state, similar to the 'undo' command found in many common appli-

\section{Results and discussions}

The proposed method was tested on both synthetic images (Section 3.1) and real medical image data (Section 3.2) to demonstrate its capabilities. The application's performance during these tasks was quantified based on the three main recommended criteria for semi-automatic segmentation [2]. To report accuracy and reproducibility measurements, Dice similarity (voxel agreement) $C_{\text {Dice }}=2 \operatorname{vol}_{\operatorname{sim}} /\left(\operatorname{vol}_{\mathrm{A}}+\operatorname{vol}_{\mathrm{B}}\right)$ was used, where $C_{\text {Dice }}$ is the Dice similarity coefficient. vol $_{\text {sim }}$ is the sum of the voxels at the intersection between trial $\mathrm{A}$ and trial $\mathrm{B}$, and $\mathrm{vol}_{\mathrm{A}}$ and $\mathrm{vol}_{B}$ represent the sum of the voxels in trials A and B, respectively. The Dice similarity coefficients were then averaged over all trials. Since our 3D Livewire method is deterministic and produces identical results given the same input contours, we measured reproducibility with different userguided contours and seedpoints as input because not all operators will choose the same slices nor will they choose the same locations for seedpoints. The orientation of the 3D segmentation and the human operator were kept constant. Efficiency was calculated by comparing the time required for our technique to segment a 3D volume to the total time needed for performing $2 \mathrm{D}$ Livewire on each slice. Due to poor image quality or user mistakes, contour errors may occur with 3D Livewire; thus, the time it takes to correct such errors is included in the time measurements as well. Finally, algorithm robustness to increasing levels of additive white Gaussian noise (AWGN) as 


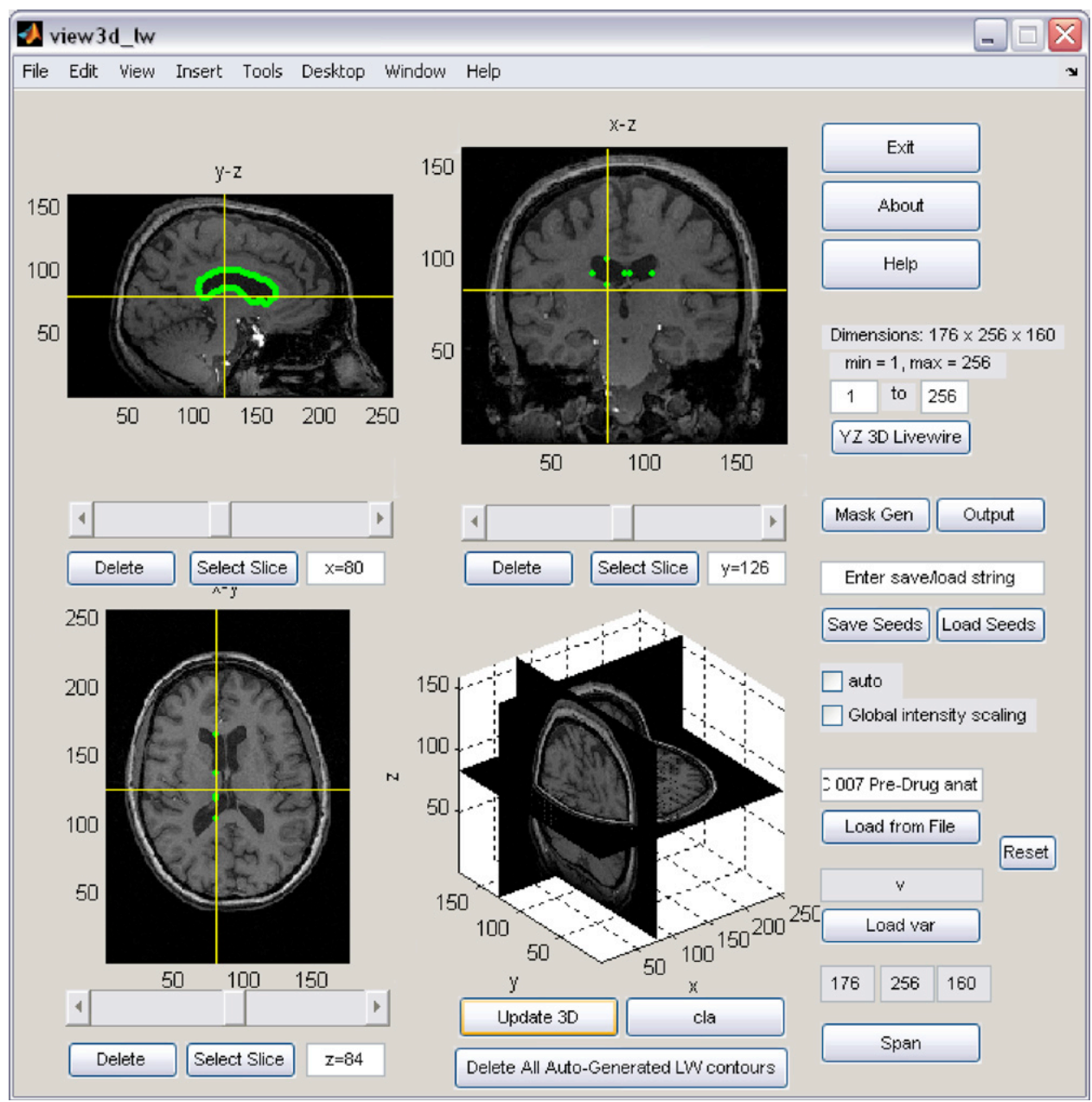

Fig. 7. Screen-capture of the proposed segmentation tool's graphical user interface during a segmentation task. Completed 2D contours are displayed in green for the three orthogonal views, providing feedback on segmentation accuracy throughout the segmentation task. Yellow lines indicate the current slice indices of the other two orientations. (For interpretation of the references to color in this figure legend, the reader is referred to the web version of the article.)

well as parameter sensitivity were also investigated in Section 3.3.

The synthetic data we used to validate our proposed method includes a mask of a left caudate nucleus (elongated object), a torus (toroidal topology object), and a fork-shaped object (branching object). We also demonstrate our method on real medical data such as the left and right ventricles from magnetic resonance imaging (T1-weighted MRI), a human vertebra (computer tomography (CT) from the Visible Human Project (VHP) [50]), and both parts of the human pelvis (CT, also from VHP).

\subsection{Synthetic data segmentation}

For the caudate nucleus (Fig. 8(a)-(c)), very few user-guided contours are required to segment the body, and additional contours at the tail guarantees an accurate delineation. The torus example (Fig. 8(d)-(f)) highlights our technique's ability to segment objects with non-spherical topology. For this scenario, only eight user-guided Livewire contours are needed. If another orientation is chosen, only six user-guided contours would be needed. For the fork-shaped object (Fig. 8(g)-(i)), only 5 user-guided
Livewire contours were required to automatically generate 209 contours. The segmentation shows a smooth transition at the branching site. Table 1 summarizes our method's accuracy and reproducibility rates, averaged over multiple trials. Table 2 shows the efficiency of our method for each computing phase. Our results show that our method is able to achieve these complex segmentation tasks in roughly $13 \%$ of the time it takes to delineate all slices using 2D Livewire.

Table 1

Reproducibility and accuracy results of our proposed method, on both synthetic and real medical image data

\begin{tabular}{lll}
\hline & Reproducibility $(\%)$ & Average accuracy $(\%)$ \\
\hline Caudate & $98.7 \pm 0.5$ & $98.5 \pm 0.2$ \\
Torus & $96.8 \pm 1$ & $96.4 \pm 0.3$ \\
Fork & $97.5 \pm 0.8$ & $97.2 \pm 0.9$ \\
Ventricles & $96.1 \pm 1.1$ & \\
Vertebra & $94.3 \pm 1.4$ & \\
Pelvis & $95.2 \pm 2.6$ & \\
\hline
\end{tabular}

Each entry in the table is the average over five trials with the corresponding standard deviation. For the ventricles, vertebra, and pelvis examples, expert manual segmentations were not available. 


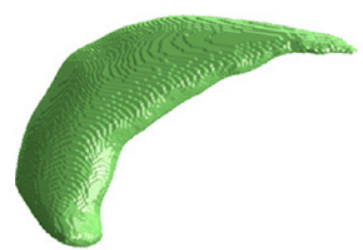

(a)

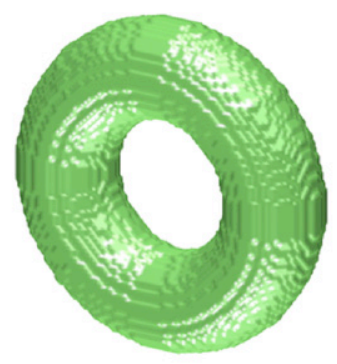

(d)

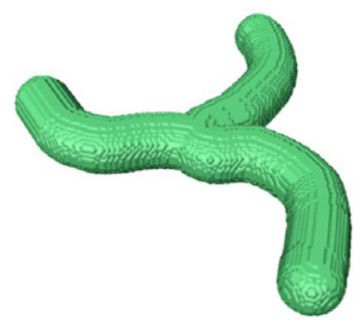

(g)

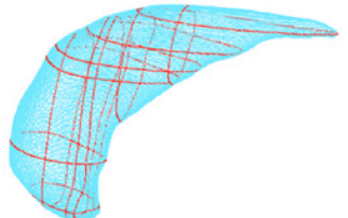

(b)

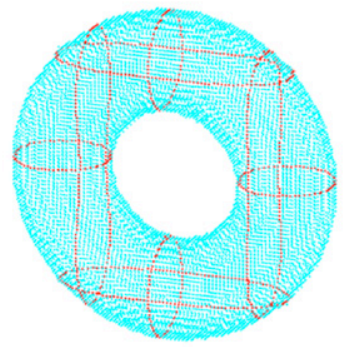

(e)

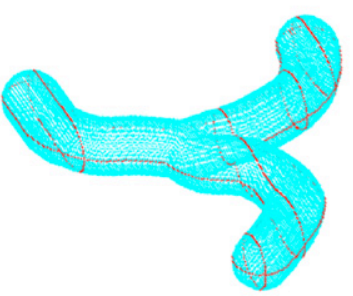

(h)

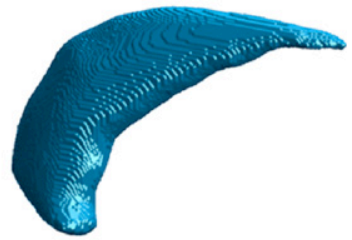

(c)

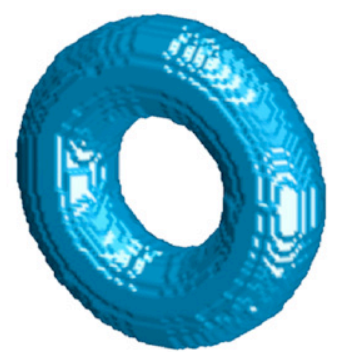

(f)

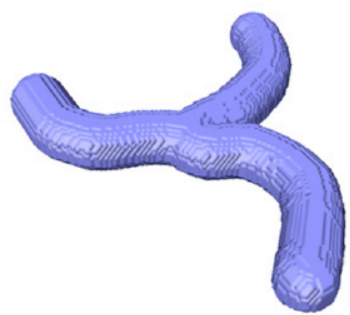

(i)

Fig. 8. Results of our proposed segmentation method on synthetic data. (a, $\mathrm{d}$ and g) Rendering of a left caudate mask, torus, and fork object, respectively. (b, e and $\mathrm{h}$ ) 3D plot of user-guided contours (red) and automatically generated contours (light blue). (c, $f$ and i) Surface renderings of the segmented synthetic examples above, using our proposed approach. (For interpretation of the references to color in this figure legend, the reader is referred to the web version of the article.)

We found that the reproducibility rates for these images are high because the input contours were computed using 2D Livewire, which has high reproducibility [33]. The minor differences between each trial largely depend on the accuracy of the chosen seedpoints. In terms of efficiency, total processing time naturally increases for volumes with high shape complexity. This is because more user-guided Livewire contours are needed to fully characterize the object and connect the various turtle map tracks together into valid maps. As the number of user-guided contours increases, so does the total amount of intersection points found on each unseen slice and ultimately, computation time. However, this higher processing time is coun- terbalanced by the fact that manual tracing of complex objects requires more user attention and segmentation time for an accurate delineation. We found that scaling a volume did not affect the number user-guided Livewire contours needed, as the same amount of these contours can still create valid turtle maps for all slices.

\subsection{Real medical data}

The first example presented here is a pair of ventricles segmented from an MRI volume (Fig. 9(a)-(c)). Here, both disjoint structures were segmented during the same task, using a total of

Table 2

Task time reduction, in seconds, achieved by our proposed method compared to performing 2DLW on each slice

\begin{tabular}{lllllll}
\hline & (I) User & (II) Auto & (III) Fix & (IV) Total & (V) 2DLW & (VI) Fraction (\%) \\
\hline Caudate & $121.2 \pm 11.4$ & $21.8 \pm 1.3$ & 0 & $143 \pm 12.6$ & $1242.6 \pm 155.2$ & $11.5 \pm 1.8$ \\
Torus & $72 \pm 9.3$ & $13.6 \pm 1.5$ & 0 & $85.6 \pm 9$ & $129 \pm 8.8$ & $1128.4 \pm 51.5$ \\
Fork & $91.8 \pm 8.7$ & $37.2 \pm 1.8$ & 0 & 0 & $629.2 \pm 50.8$ & $3862.6 \pm 230.2$ \\
Ventricles & $556.2 \pm 54.5$ & $73 \pm 5.5$ & $38.4 \pm 22.3$ & $836.2 \pm 41.1$ & $4283.2 \pm 230.9$ & $11.5 \pm 1.2$ \\
Vertebra & $741.8 \pm 32.5$ & $56 \pm 4$ & $126.8 \pm 56.7$ & $1047.8 \pm 55$ & $4626.6 \pm 237$ \\
Pelvis & $742.4 \pm 16$ & $178.6 \pm 18.4$ & $0.5 \pm 0.4$ & $22.7 \pm 1.5$ \\
\hline
\end{tabular}

Each step for the examples is averaged over five trials. Standard deviation values between each set of trials are included. (I) User-interaction time with our tool. (II) Automatic processing time of our tool. (III) Time required for manual corrections. (IV) Total task time of our tool. (V) Task time using 2DLW on all slices. (VI) Fraction of time (\%) required for our tool compared to 2DLW on all slices (IV)/(V). 
(a)

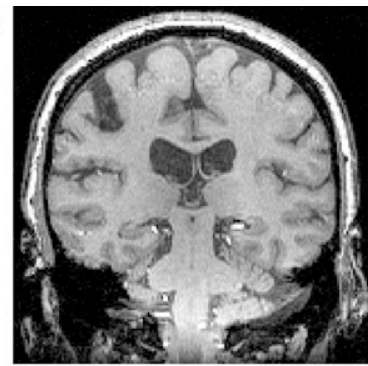

(d)

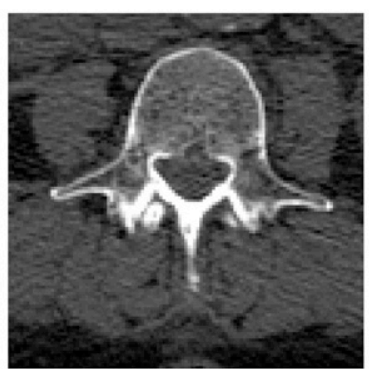

(g)

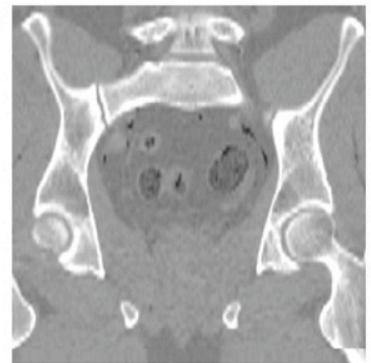

(b)

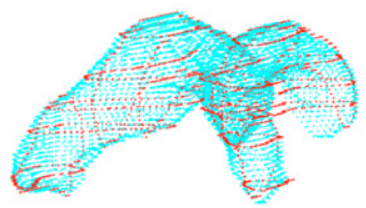

(e)

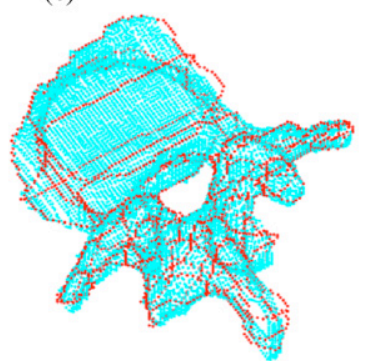

(h)

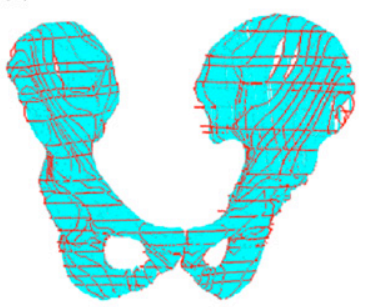

(c)

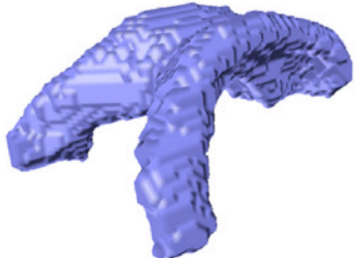

(f)

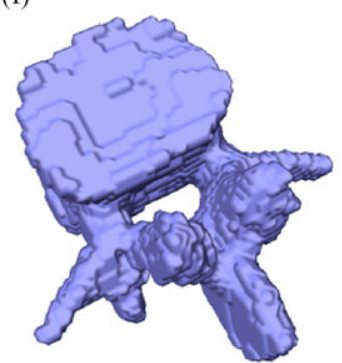

(i)

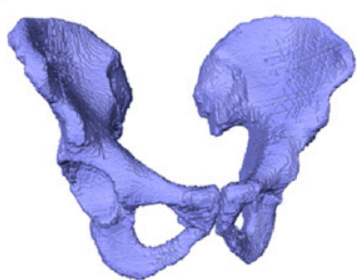

Fig. 9. Results of our proposed segmentation method on real 3D medical data. (a, $d$ and g) Original 3D images of a human brain (T1-MRI), spine (CT), and pelvic region (CT), respectively. (b, e and h) 3D plots of user-guided contours (red) and automatically generated contours (light blue). Twenty-four (red) used to segment 200 (cyan), 17 to segment 88, and 80 to segment 277, respectively. (c, $f$ and i) Surface renderings of the segmented examples above, using our proposed method. (For interpretation of the references to color in this figure legend, the reader is referred to the web version of the article.)

24 input contours to automatically segment 105 slices (approximately 200 contours). In order to accurately capture the tail regions, a higher concentration of input contours was provided there. All object features were successfully captured, including the separation. Next, a vertebra was extracted from the male CT scan of the VHP [50] (Fig. 9(d)-(f)). The human vertebra is toroidal, with pronounced protrusions. Also, the volume contains multiple vertebrae, and parts of two vertebrae often appear on the same slice. Here, our proposed method successfully extracted the vertebra using 17 input contours to segment 88 slices. Lastly, the human pelvis (Fig. 9(g)-(i)), also from the CT scan of the VHP, was segmented using 80 input contours to segment 277 slices. For this example, due to the very thin bone characteristics at the ilium, the minimal number of input
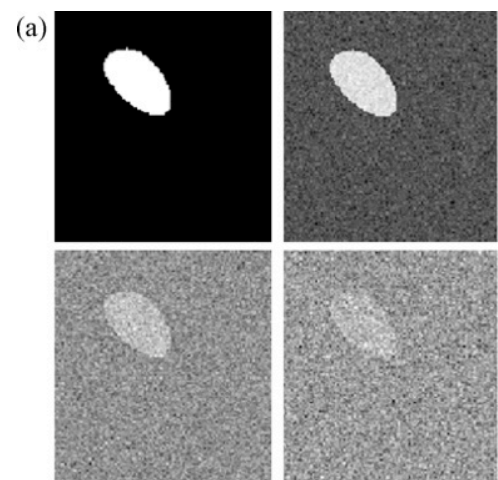

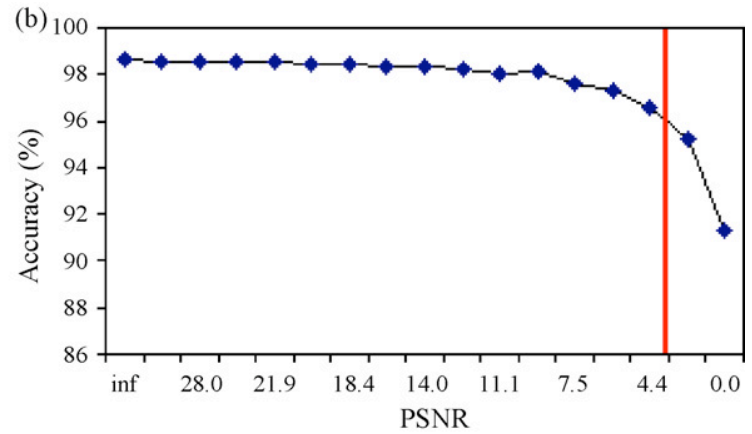

Fig. 10. Our proposed method's performance reflected in segmentation accuracy as AWGN is progressively added. (a) Slices of a left caudate mask with increasing noise and PSNR levels of $\infty, 20.0,6.0$, and 0. (b) Red line denotes the PSNR value corresponding to 96\% accuracy, before which accuracy level stays consistently high. Very high noise levels obscures the ability of the user to choose reliable Livewire seedpoints, thus affecting overall accuracy. (For interpretation of the references to color in this figure legend, the reader is referred to the web version of the article.) 
contours to create correct turtle maps at this area is difficult to achieve. This results in minor gaps in the segmentation, but these gaps were easily fixed with our tool using 2D Livewire to overwrite these problematic slices. The above examples were tested for reproducibility and efficiency over non-expert manual segmentation, as shown in Tables 1 and 2. Similar to the synthetic data experiments, our proposed method is shown to be much more efficient than performing 2D Livewire on every slice in a real medical image and the segmentation is highly reproducible when provided different input contours.

\subsection{Algorithm robustness and parameter sensitivity}

Using consistent user-defined seedpoints for the user-guided contours, the caudate mask volume was subjected to incremental levels of AWGN and then segmented using our proposed method. Accuracy levels were then plotted against the peak signal to noise ratio (PSNR) of the volume, defined as PSNR $=20 \log _{10}\left(\max (\right.$ object intensity $\left.) / \sigma_{\text {noise }}\right)$. The segmentation results are shown in Fig. 10(a) and accuracy results are shown in Fig. 10(b). As expected, the accuracy level decreases as the increasing amount of noise corrupts the image, but our method is able to recover much of the object even under high amounts of noise.

Parameter selection was not a problem in obtaining accurate results, and our implementation uses equal weighting for each term in $(1)\left(w_{1 \ldots 5}=1\right)$. Nevertheless, we investigated the effect of parameter sensitivity on the synthetic examples in Fig. 8 to determine the change in accuracy when varying each weight value. We found that varying each weight by as much as $\pm 50 \%$ did not change the accuracy by more than $3.1 \%$ in the test datasets.

\section{Conclusions}

This paper presented a novel, highly automated 3D Livewirebased segmentation technique which can not only seamlessly handle arbitrarily complex shape topologies commonly found in medical images, but also does so with minimal user supervision. Also, an intuitive segmentation tool based on this framework was presented, which features easy transitions between 2D and 3D Livewire modes, facilitates efficient correction of user mistakes, and provides $3 \mathrm{D}$ visualization of the result. Our proposed method was shown to successfully and robustly segment a variety of complex synthetic objects and real anatomical structures with branchings, concavities/protrusions, and non-spherical topologies. Tests of our method on real $3 \mathrm{D}$ medical data show a high degree of segmentation reproducibility (up to $96.1 \%$ ) and task time reduction (up to $83.7 \%$ reduction when compared to using 2D Livewire on each slice).

Development of this Livewire-based, highly automated framework is ongoing. Enhancing algorithm robustness to user-mistakes is the primary focus, and we are refining the segmentation tool's user interface to be even more user-friendly. Also, our proposed algorithm is being improved to achieve an even higher efficiency.

\section{References}

[1] Rajapakse J, Kruggel F. Segmentation of MR images with intensity inhomogeneities. Image and Vision Computing 1998;16:165-80.

[2] Olabarriaga S, Smeulders A. Interaction in the segmentation of medical images: a survey. Medical Image Analysis 2001;5:127-42.

[3] Kang Y, Engelke K, Kalender W. Interactive 3D editing tools for image segmentation. Medical Image Analysis 2004;8:35-46.

[4] McInerney T, Terzopoulos D. Deformable models in medical image analysis: a survey. Medical Image Analysis 1996;1:91-108.

[5] Kass M, Witkin A, Terzopoulos D. Snakes: active contour models. International Journal of Computer Vision 1988;1:321-31.

[6] McInerney T, Terzopoulos D. T-snakes: topology adaptive snakes. Medical Image Analysis 2000;4:73-91.

[7] Delingette H. General object reconstruction based on simplex meshes. IJCV 1999;32:111-46.

[8] Lachaud J-O, Montanvert A. Deformable meshes with automated topology changes for coarse-to-fine 3D surface extraction. Medical Image Analysis 1998;3(2):187-207.

[9] Montagnat J, Delingette H, Ayache N. A review of deformable surfaces: topology, geometry and deformation. Image and Vision Computing 2001;19(14):1023-40.

[10] Liang J, McInerney T, Terzopoulos D. United snakes. Medical Image Analysis 2006;10:215-33.

[11] Osher S, Paragios N. Geometric level set methods in imaging vision and graphics. Springer-Verlag; 2003.

[12] Caselles V, Kimmel R, Sapiro G. Geodesic active contours. IJCV 1997;22(1):61-79.

[13] Sethian JA. Level set methods; evolving interfaces in geometry, fluid mechanics. Cambridge University Press; 1996.

[14] Bresson X, Esedoglu S, Vandergheynst P, Thiran J-P, Osher S. Fast global minimization of the active contour/snake model. Journal of Mathematical Imaging and Vision 2007;28(2):151-67.

[15] Cohen L, Kimmel R. Global minimum for active contour models: a minimal path approach. IJCV 1997;24:57-78.

[16] Paragios N. MICCAI 2003:678-86.

[17] Rumpf M, Strzodka R. Level set segmentation in graphics hardware. IEEE International Conference on Image Processing 2001:1103-6.

[18] Lefohn A, Cates J, Whitaker R. MICCAI 2003:564-72.

[19] Yushkevich P, Piven J, Hazlett H, Smith R, Ho S, Gee J, et al. User-guided 3D active contour segmentation of anatomical structures: significantly improved efficiency and reliability. NeuroImage 2006;31:1116-28.

[20] McInerney T, Akhavan S. ISBI 2006:398-401.

[21] Urschler M, Mayer H, Bolter R, Leberl F. The livewire approach for the segmentation of left ventricle electron-beam CT images. In: 26th workshop of the Austrian association for pattern recognition (AGM/AAPR). 2002.

[22] Gougoutas A, Wheaton A, Borthakur A, Shapiro E, Kneeland J, Udupa J, et al. Cartilage volume quantification via live wire segmentation. Academic Radiology 2004;11(January):1389-95.

[23] Zhang H, Nosher J, Yim P. SPIE Medical Imaging 2006:98-104.

[24] Jackowski M, Satter M, Goshtasby A. Approximating digital 3D shapes by rational gaussian surfaces. IEEE Transactions on Visualization and Computer Graphics 2003;9(1):56-69.

[25] Zhao H, Osher S, Merriman B, Kang M. Implicit, nonparametric shape reconstruction from unorganized points using a variational level set method. Computer Vision and Image Understanding 2000;80:295-319.

[26] Turk G, O'Brien J. Shape transformation using variational implicit functions. In: Proceedings of the SIGGRAPH 99: Computer Graphics. 1999. p. $335-42$.

[27] Hoppe H, DeRose T, Duchamp T, McDonald J, Stuetzle W. Surface reconstruction from unorganized points. In: SIGGRAPH '92: Proceedings of the 19th annual conference on computer graphics and interactive techniques. New York, NY, USA: ACM Press; 1992. p. 71-8.

[28] Saboo R, Rosenman J, Pizer S. Geointerp: contour interpolation with geodesic snakes. The Insight Jounal 2006.

[29] Boykov Y, Jolly M. MICCAI 2000:276-86.

[30] Boykov Y, Funka-Lea G. Graph cuts and efficient N-D image segmentation. International Journal of Computer Vision 2006;70:109-31 
[31] Rother C, Kolmogorov V, Blake A. GrabCut: interactive foreground extraction using iterated graph cuts. ACM Transactions on Graphics 2004:309-14.

[32] Grady L. Random walks for image segmentation. IEEE Transactions Pattern Analysis and Machine Intelligence 2006;28(November): 1768-83.

[33] Barrett W, Mortensen E. Interactive live-wire boundary extraction. Medical Image Analysis 1997;1:331-41.

[34] Chodorowski A, Mattsson U, Langille M, Hamarneh G. Color lesion boundary detection using live wire. SPIE Medical Imaging 2005:1589-96.

[35] Falcao A, Udupa J, Samarasekera S, Sharma S, Hirsch B, Lotufo R. Usersteered image segmentation paradigms: live wire and live lane. Graphical Models and Image Processing 1998;60:233-60.

[36] Falcao A, Udupa J, Miyazawa F. An ultra-fast user-steered image segmentation paradigm: live wire on the fly. IEEE TMI 2000;19(January):55-62.

[37] Kang HW, Shin SY. Enhanced lane: interactive image segmentation by incremental path map construction. Graphical Models 2002;64(5):282-303.

[38] Schenk A, Prause G, Peitgen H. Local cost computation for efficient segmentation of 3D objects with live wire. SPIE Medical Imaging 2001:1357-64.

[39] Souza A, Udupa J, Grevera G, Sun DOY, Suri N. Iterative live wire and live snake: new user-steered 3D image segmentation paradigms. SPIE Medical Imaging 2006;(March):1159-65.

[40] Schenk A, Prause G, Peitgen H. Efficient semiautomatic segmentation of 3D objects in medical images. MICCAI 2000:186-95.

[41] Malmberg F, Vidholm E, Nystrom I. A 3D Live-Wire Segmentation Method for Volume Images Using Haptic Interaction.

[42] Falcao A, Bergo F. Interactive volume segmentation with differential image foresting transforms. IEEE TMI 2004;23:1100-8.

[43] Falcao A, Udupa J. A 3D generalization of user-steered live-wire segmentation. Medical Image Analysis 2000;4:389-402.

[44] Lu K, Higgins W. Improved 3D live-wire method with application to 3D CT chest image analysis. SPIE Medical Imaging 2006:189-203.

[45] Hamarneh G, Yang J, McIntosh C, Langille M. 3D live-wire-based semi-automatic segmentation of medical images. SPIE Medical Imaging 2005:1597-603.

[46] Papert S. Mindstorms: children, computers, and powerful ideas. New York, NY, USA: Basic Books, Inc.; 1980

[47] Poon M, Hamarneh G, Abugharbieh R. Segmentation of complex objects with non-spherical topologies from volumetric medical images using $3 \mathrm{D}$ livewire. SPIE Medical Imaging 2007;6512-31:1-10.
[48] Canny J. A computational approach to edge detection. IEEE Transactions Pattern Analysis and Machine Intelligence 1986;8(6):679-98.

[49] Dijkstra E. A note on two problems in connexion with graphs. In Numerical Mathematik 1959;1:269-70.

[50] Ackerman M. The visible human project. Proceedings of the IEEE 1998;86:504-11.

Miranda Poon received her Bachelor's and Master's degrees in applied science from the Department of Electrical and Computer Engineering at the University of British Columbia, Canada, in 2005 and 2008, respectively. Her research interests focus on interactive segmentation techniques and multi-dimensional image processing.

Ghassan Hamarneh received his B.Sc. degree from the Department of Electrical Engineering, Jordan University, in 1995, and M.Sc. degree with distinction in digital communications from the Chalmers University of Technology in 1997 Before joining the School of Computing Science, Simon Fraser University, Burnaby, BC, Canada, as an Assistant Professor in 2003, he was a Postdoctoral Fellow at the Hospital for Sick Children, Mouse Imaging Centre, and the Department of Medical Biophysics, University of Toronto, Toronto, ON, Canada (2001-2003). He completed his doctoral studies (2001) at the Department of Signals and Systems, Chalmers University of Technology, and as a Predoctoral Research Fellow (2000-2001) at the Department of Computer Science, University of Toronto. $\mathrm{He}$ is the co-founder (2003) and co-director of the Medical Image Analysis Laboratory (MIAL), Simon Fraser University. His main research interests are medical image processing, segmentation and registration, and anatomical shape modelling and analysis.

Rafeef Abugharbieh completed her doctoral studies at the Department of Signals and Systems at Chalmers University of Technology in Goteborg, Sweden (2001). She received her Master's degree with distinction in digital communications from Chalmers University (1997) and her Bachelor's degree in electrical engineering from the Department of Electrical Engineering at Jordan University in Amman, Jordan (1995). She is an IEEE member and a member of the IEEE Engineering in Medicine and Biology Society (EMBS) since 2003. She is also an associate founding member of the IEEE EMBS Vancouver Section (2004). Dr. Abugharbieh joined the Department of Electrical and Computer Engineering at the University of British Columbia as an Assistant Professor in 2004 where she co-founded (2005) and co-directs the Biomedical Signal and Image Computing Laboratory (BiSICL), a multi-disciplinary research lab dedicated to computational research in biomedical applications. Her main research interests are in the areas of image processing and analysis particularly in medical imaging applications. Homepage: http://www.ece.ubc.ca/ rafeef/. 